University of Wollongong

Research Online

Faculty of Informatics - Papers (Archive)

Faculty of Engineering and Information

Sciences

$11-9-2005$

\title{
Generalized Williamson and Wallis-Whiteman constructions for improved square order-8 CO STBCs
}

\author{
Le Chung Tran \\ University of Wollongong, Ictran@uow.edu.au \\ Tadeusz A. Wysocki \\ University of Wollongong, wysocki@uow.edu.au \\ Jennifer Seberry \\ University of Wollongong, jennie@uow.edu.au
}

A. Mertins
University of Wollongong, mertins@uow.edu.au
Sarah A. Spence
Franklin Olin College of Engineering, Needham USA

Follow this and additional works at: https://ro.uow.edu.au/infopapers

Part of the Physical Sciences and Mathematics Commons

\section{Recommended Citation}

Tran, Le Chung; Wysocki, Tadeusz A.; Seberry, Jennifer; Mertins, A.; and Spence, Sarah A.: Generalized Williamson and Wallis-Whiteman constructions for improved square order-8 CO STBCs 2005.

https://ro.uow.edu.au/infopapers/277

Research Online is the open access institutional repository for the University of Wollongong. For further information contact the UOW Library: research-pubs@uow.edu.au 


\title{
Generalized Williamson and Wallis-Whiteman constructions for improved square order-8 CO STBCs
}

\author{
Abstract \\ Constructions of square, maximum rate Complex Orthogonal Space-Time Block Codes (CO STBCs) are \\ well known, however codes constructed via the known methods include numerous zeros, which impede \\ their practical implementation. By modifying the Williamson and Wallis-Whiteman arrays to apply to \\ complex matrices, we propose two methods of construction of square, order- $4 \mathrm{n}$ CO STBCs from square, \\ order-n codes which satisfy certain properties. Applying the proposed methods, we construct square, \\ maximum rate, order-8 CO STBCs with no zeros, such that the transmitted symbols equally disperse \\ through transmit antennas. Those codes, referred to as the improved square CO STBCs, have the \\ advantages that the power is equally transmitted via each transmit antenna during every symbol time slot \\ and that a lower peak-to-mean power ratio per each antenna is required to achieve the same bit error \\ rates as for the conventional CO STBCs with zeros.

\section{Disciplines} \\ Physical Sciences and Mathematics

\section{Publication Details} \\ This article was originally published as Tran, L, Wysock, TA, Seberry, J et al, Generalized Williamson and \\ Wallis-Whiteman constructions for improved square order-8 CO STBCs, In B. Walke, K. David, Mhaardt and \\ P. Mahonen (Eds.), Proceedings of the 16th International Symposium on Personal Indoor and Mobile \\ Radio Communications (pp. 1155-1159), IEEE PIMRC 2005, Berlin Germany, 11-15 September 2005. \\ Germany: IEEE. Copyright IEEE 2005.
}




\section{Generalized Williamson and Wallis-Whiteman Constructions for Improved Square Order-8 CO STBCs}

\author{
Le Chung Tran, Tadeusz A. Wysocki, and \\ Jennifer Seberry \\ School of Elec.,Comp. \& Telecom. Eng. \\ University of Wollongong \\ Wollongong, NSW 2522, Australia \\ Email: lct71,wysocki,jennie@uow.edu.au
}

Alfred Mertins

Institute of Physics

University of Oldenburg

26111 Oldenburg, Germany

Email: alfred.mertins@uni-oldenburg.de

\author{
Sarah A. Spence \\ Franklin Olin \\ College of Engineering \\ Needham, MA 02492-1245, USA \\ Email: sarah.spence@olin.edu
}

\begin{abstract}
Constructions of square, maximum rate Complex Orthogonal Space-Time Block Codes (CO STBCs) are well known, however codes constructed via the known methods include numerous zeros, which impede their practical implementation. By modifying the Williamson and Wallis-Whiteman arrays to apply to complex matrices, we propose two methods of construction of square, order- $4 n$ CO STBCs from square, order$n$ codes which satisfy certain properties. Applying the proposed methods, we construct square, maximum rate, order-8 CO STBCs with no zeros, such that the transmitted symbols equally disperse through transmit antennas. Those codes, referred to as the improved square $C O S T B C s$, have the advantages that the power is equally transmitted via each transmit antenna during every symbol time slot and that a lower peak-to-mean power ratio per each antenna is required to achieve the same bit error rates as for the conventional CO STBCs with zeros.
\end{abstract}

\section{INTRODUCTION}

Complex Orthogonal Space-Time Block Codes (CO STBCs) have been intensively examined, as they provide large transmit diversity and increase the capacity of wireless channels, while requiring a very simple Maximum Likelihood (ML) decoding method [1], [2], [3] [4] [5]. A $p \times n$ CO STBC over $k$ variables is corresponding to $n$ transmit antennas, decoding delay (or memory length) of $p$, and rate $R=\frac{k}{p}$. Given $n$ and $R$, the goal is to minimize the decoding delay $p$. Hence, square CO STBCs are particularly interesting because they require the minimum processing delay (minimum memory length as well) for the same rate and the same number of transmit antennas. Another consideration for practical implementation is the number of zeros in a code. Compared to a code with fewer zeros, a code with more zeros results in a higher peak-tomean power ratio for the transmit antennas to achieve the same Bit Error Rates (BER). Having many zeros can also impede practical implementation since some transmit antennas must be turned off during transmission. Turning transmit antennas off during transmission is inconvenient, especially in high data rate wireless communication systems. Furthermore, it would be more practical if the power of signals can be equally transmitted via each transmit antenna during every symbol time slot. Given the above considerations for CO STBCs, this paper focuses on constructing square CO STBCs with maximum rate, minimum decoding delay, no zero entries, and equal power transmission per transmit antenna during each symbol time slot.

The simplest square CO STBCs is the Alamouti code [1], which achieves a rate one for two transmit antennas. In contrast, square CO STBCs for more than two transmit antennas cannot achieve rate one [2], [6], but they can still achieve full diversity for a given number of transmit antennas. Constructions of square CO STBCs for a higher number of transmit antennas, e.g. 4 and 8, have been well examined in literature, such as [2] and [5]. These structures yield square CO STBCs of maximum rate, which is, for instance, $1 / 2$ for 8 transmit antennas. However, these maximum rate codes have many zero entries, which are undesirable.
It is important to clarify that, according to Liang's paper [2], the maximum achievable rate for CO STBCs of orders $n=2 m-1$ or $n=2 m$ is (see Eq. (130) in [2]):

$$
R_{\max }=(m+1) / 2 m
$$

However, note that this maximum rate is only achievable for rectangular constructions, except for the special case when $m=1$, i.e. when $n=1$ or $n=2$. For square constructions of orders $n=2^{a}(2 b+1)$, the maximum achievable rate is:

$$
R_{\max }=(a+1) / 2^{a}(2 b+1)
$$

When $m=1$, (1) and (2) provide the same results. Readers should refer to Corollary 2 and Section II D in [2], or Section IV in [5] for more details.

Particularly, for $n=8$, i.e., $m=4, a=3$ and $b=0$, the maximum achievable rate of rectangular CO STBCs following (1) is $5 / 8$, while the maximum achievable rate of square $\mathrm{CO}$ STBCs according to (2) is 1/2 only. In Liang's paper, the authors made an unclear statement in the abstract that the achievable maximum rate for $n=2 m-1$ and $n=2 m$ is $(m+1) / 2 m$, but did not state if this maximum rate is achievable by rectangular or square constructions. This easily makes readers confused, except when readers go deeply into the Liang's paper.

Square CO STBCs have a great advantage over rectangular CO STBCs that they require a much smaller length of the codes, i.e., much smaller processing delay, with the consequence of the slightly smaller maximum code rate compared to the achievable maximum code rate of rectangular CO STBCs. Let us consider CO STBCs for $n=8$ transmit antennas as an example. The rectangular $\mathrm{CO}$ STBC that achieves the maximum rate $5 / 8$ requires the length of 112 symbol time slots. The $[112,8,70]$ CO STBC given in Appendix E in Liang's paper [2] is an example for this case. As opposite to rectangular $\mathrm{CO}$ STBCs, square $\mathrm{CO}$ STBCs only require the length of 8 symbol time slots to achieve the maximum rate $1 / 2$, which is slightly smaller than the maximum rate of rectangular CO STBCs. Clearly, square CO STBCs require a much shorter length, especially for a large number of transmit antennas, with the consequence of a slightly lower maximum code rate. For this reason, in this paper, we only consider square CO STBCs.

Square CO STBCs with no zero entries have been proposed in the literature, such as [1] and [4], for orders 2, 4. In [7], we constructed two square, order $8 \mathrm{CO}$ STBCs with fewer zeros than the conventional codes [2], [5]. Later, in [8] and [9], we constructed a square, order 8 CO STBC $\mathbf{Z}$ without any zero which is given in (3).

As pointed out in [8], the entries $z_{l k}(l=5, \ldots, 8$, $k=1, \ldots, 8)$ of $\mathbf{Z}$ are composed of the real part of one indeterminate and the imaginary part of another indeterminate, e.g., $z_{51}=-s_{4}^{R}+i s_{3}^{I}$. This observation means that if the indeterminates $s_{1}, \ldots, s_{4}$ are chosen from the complex signal constellations where $s_{j}^{R}$ or $s_{j}^{I}(j=1 \ldots 4)$ can be equal to 


$$
\mathbf{Z}=\left[\begin{array}{rrrrrrrr}
s_{1} & s_{1} & s_{2} & s_{2} & s_{3} & s_{4} & s_{3} & s_{4} \\
s_{1} & -s_{1} & s_{2} & -s_{2} & s_{4}^{*} & -s_{3}^{*} & s_{4}^{*} & -s_{3}^{*} \\
s_{2}^{*} & s_{2}^{*} & -s_{1}^{*} & -s_{1}^{*} & s_{3} & s_{4} & -s_{3} & -s_{4} \\
s_{2}^{*} & -s_{2}^{*} & -s_{1}^{*} & s_{1}^{*} & s_{4}^{*} & -s_{3}^{*} & -s_{4}^{*} & s_{3}^{*} \\
-s_{4}^{R}+i s_{3}^{I} & -s_{3}^{R}+i s_{4}^{I} & -s_{4}^{R}+i s_{3}^{I} & -s_{3}^{R}+i s_{4}^{I} & s_{2}^{R}-i s_{1}^{I} & s_{2}^{R}-i s_{1}^{I} & s_{1}^{R}-i s_{2}^{I} & s_{1}^{R}-i s_{2}^{I} \\
-s_{3}^{R}-i s_{4}^{I} & s_{4}^{R}+i s_{3}^{I} & -s_{3}^{R}-i s_{4}^{I} & s_{4}^{R}+i s_{3}^{I} & s_{2}^{R}-i s_{1}^{I} & -s_{2}^{R}+i s_{1}^{I} & s_{1}^{R}-i s_{2}^{I} & -s_{1}^{R}+i s_{2}^{I} \\
-s_{4}^{R}+i s_{3}^{I} & -s_{3}^{R}+i s_{4}^{I} & s_{4}^{R}-i s_{3}^{I} & s_{3}^{R}-i s_{4}^{I} & s_{1}^{R}+i s_{2}^{I} & s_{1}^{R}+i s_{2}^{I} & -s_{2}^{R}-i s_{1}^{I} & -s_{2}^{R}-i s_{1}^{I} \\
-s_{3}^{R}-i s_{4}^{I} & s_{4}^{R}+i s_{3}^{I} & s_{3}^{R}+i s_{4}^{I} & -s_{4}^{R}-i s_{3}^{I} & s_{1}^{R}+i s_{2}^{I} & -s_{1}^{R}-i s_{2}^{I} & -s_{2}^{R}-i s_{1}^{I} & s_{2}^{R}+i s_{1}^{I}
\end{array}\right]
$$

zero, e.g., the QPSK constellation $(1,-1, i,-i)$ then, some of the entries of the matrix $\mathbf{Z}$ can be equal to zero depending on the transmitted data. Therefore, such constellations should be avoided. An example of the constellation where the power is evenly spread among the Tx antennas independently of the transmitted data is the QPSK constellation $(1+i, 1-i,-1+i,-1-i)$.

The square CO STBC in (3) has the following advantages:

1) It is not required to turn off any transmit antenna during transmission, unlike in the conventional CO STBC [2], [5].

2) When the indeterminates are chosen from a suitable constellation, $\mathbf{Z}$ has no zero entries, hence, it requires a smaller peak power per Tx antenna to achieve the same BER as in the conventional square CO STBCs with zeros [2], [5]. Equivalently, it provides a better BER compared to the conventional square CO STBCs with the same peak power at transmit antennas.

Independently, from Amicable Orthogonal Designs (AODs), C. Yuen et al. [10] constructed the following solitary, square, order-8 CO STBC with no zeros:

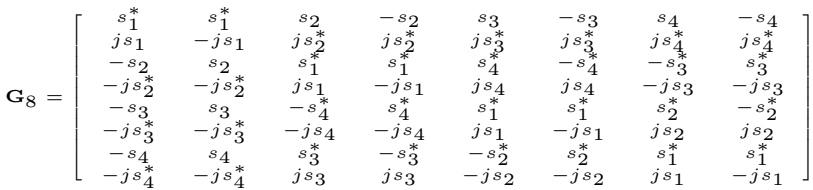
(4)

where $j=\sqrt{-1}$. The background knowledge on AODs can be found in [11]. This square CO STBC has an advantage over our code $\mathbf{Z}$ in that it does not require the restriction on signal constellations. However, from amicable orthogonal designs, it is difficult to construct square CO STBCs, especially for those codes of high orders, since we have to incorporate many weighting matrices. For instance, to construct a square, maximum rate CO STBC of order 8 , we have to find 8 matrices of size $8 \times 8$ (4 weighting matrices for the real parts of variables and 4 other weighting matrices for the imaginary parts) which simultaneously satisfy several strong conditions of AODs [11], [12], [10].

In this paper, by modifying the Williamson and WallisWhiteman arrays to apply to complex matrices, we propose two novel methods of construction of square, order- $4 n \mathrm{CO}$ STBCs from square, order- $n$ codes which satisfy certain properties. Applying the proposed methods, we construct square, maximum rate, order- $8 \mathrm{CO}$ STBCs with no zeros, such that the transmitted symbols equally disperse through transmit antennas. Besides having the maximum rate, the minimal decoding delay, and no zero entries, the resultant codes, referred to as the improved square CO STBCs, have the following practical advantages: a) They do not require any restriction on allowable signal constellations; b) It is possible to transmit symbols with equal power for any STS at any transmit antenna; and c) A lower peak power per transmit antenna is required to achieve the same bit error rates as for the conventional CO STBCs with zeros.

As mentioned in more details later in this paper, in order to construct, for instance, $8 \times 8 \mathrm{CO}$ STBCs, the main task in our methods is to find two sub-matrices of size $2 \times 2$ which satisfy certain properties, rather than finding 8 weighting matrices of size $8 \times 8$ simultaneously as in the AOD approaches, such as in [10]. More importantly, our methods give a transition from square, order- $n$ CO STBCs satisfying certain properties to square, order- $4 n$ CO STBCs. Our proposed methods might even lead to the constructions of square CO STBCs of higher orders, such as 16 or 32, with fewer zeros or even without zeros.

The paper is organized as follows. In Section II, we provide definitions and notations used throughout the paper. In Section III, we propose two methods for constructing high-rate, square CO STBCs of order $N=4 n$ from sub-matrices of order $n$. In Section IV, we use the proposed methods to construct square, maximum rate, order $8 \mathrm{CO}$ STBCs, which are superior in several aspects to other known codes to date. The paper is concluded by Section V.

\section{DEFINITIONS AND NotATIONS}

Our proposed constructions in this paper are based on the following matrices, which are the variations of the Williamson and Wallis-Whiteman arrays mentioned in [11] (pp. 121 and 99, respectively), modified to apply to complex matrices:

$$
\begin{aligned}
\mathcal{O}_{1}= & {\left[\begin{array}{cccc}
\mathbf{A} & \mathbf{B} & \mathbf{C} & \mathbf{D} \\
-\mathbf{B} & \mathbf{A} & \overline{\mathbf{D}} & -\overline{\mathbf{C}} \\
-\mathbf{C} & -\overline{\mathbf{D}} & \mathbf{A} & \overline{\mathbf{B}} \\
-\mathbf{D} & \overline{\mathbf{C}} & -\overline{\mathbf{B}} & \mathbf{A}
\end{array}\right] } \\
\mathcal{O}_{2} & =\left[\begin{array}{cccc}
\mathbf{A} & \mathbf{B} & \mathbf{C} & \mathbf{D} \\
-\overline{\mathbf{B}} & \overline{\mathbf{A}} & -\mathbf{D} & \mathbf{C} \\
-\mathbf{C} & \overline{\mathbf{D}} & \mathbf{A} & -\overline{\mathbf{B}} \\
-\overline{\mathbf{D}} & -\mathbf{C} & \mathbf{B} & \overline{\mathbf{A}}
\end{array}\right]
\end{aligned}
$$

where $\overline{\mathbf{X}}$ is the matrix derived from a matrix $\mathbf{X}$ by replacing all variables in $\mathbf{X}$ by their conjugates, i.e., $\overline{\mathbf{X}}=\left(\mathbf{X}^{H}\right)^{T}$. $(.)^{H}$ denotes the Hermitian transposition while $(.)^{T}$ denotes the transposition (but not conjugate). $\mathbf{A}, \mathbf{B}, \mathbf{C}$ and $\mathbf{D}$ are $n \times n$, square, orthogonal matrices of complex variables. Hence, $\mathcal{O}_{1}$ and $\mathcal{O}_{2}$ are $4 n \times 4 n$ matrices of complex variables.

Let $\mathcal{O}$ be a general notation representing either $\mathcal{O}_{1}$ or $\mathcal{O}_{2}$. Define $N=4 n$ and present $N$ as $N=2^{a}(2 b+1)$, where $a$ and $b$ are integers. Let $\mu(N)$ be the maximum number of variables in $\mathcal{O}$. It is well known that the maximum number of variables in the square CO STBC of order $N$ is $\mu(N)=a+1$. Readers may refer to [5], [11], or Corollary 2 in [2] for more details. Let $\mu_{A}, \mu_{B}, \mu_{C}$ and $\mu_{D}$ be the number of variables in $\mathbf{A}, \mathbf{B}$, $\mathbf{C}$, and $\mathbf{D}$, respectively.

Let $U$ and $\mathcal{I}_{U}$ be the set of all variables in $\mathcal{O}$ and the set of all indices of elements in $U$, respectively. Similarly, let:

$U_{1}=\left\{s_{A 1}, s_{A 2}, \ldots, s_{A \mu_{A}}\right\} ; U_{2}=\left\{s_{B 1}, s_{B 2}, \ldots, s_{B \mu_{B}}\right\}$

$U_{3}=\left\{s_{C 1}, s_{C 2}, \ldots, s_{C \mu_{C}}\right\} ; U_{4}=\left\{s_{D 1}, s_{D 2}, \ldots, s_{D \mu_{D}}\right\}$ 
be the sets of variables in $\mathbf{A}, \mathbf{B}, \mathbf{C}$, and $\mathbf{D}$, respectively, and let $\mathcal{I}_{U_{i}}$, for $i=1 \ldots 4$, be the sets of indices of variables in the sub-matrices $\mathbf{A}, \mathbf{B}, \mathbf{C}$, and $\mathbf{D}$, respectively.

We require that the sub-matrices $\mathbf{A}, \mathbf{B}, \mathbf{C}$, and $\mathbf{D}$ satisfy:

$$
\begin{cases}\bigcup U_{i}=U & i=1, \ldots, 4 \\ \bigcap U_{i} U_{j}=\varnothing & i \neq j\end{cases}
$$

where $\varnothing$ is the empty set.

With the condition (8), clearly, if $\mathcal{O}$ comprises the maximum number of variables, we have:

$$
\mu_{A}+\mu_{B}+\mu_{C}+\mu_{D}=\mu(N)
$$

Since $\mathbf{A}$ is a matrix on variables $\left\{s_{A 1}, s_{A 2}, \ldots, s_{A \mu_{A}}\right\}$, we define the vector $\mathbf{s}_{\mathbf{A}}=\left(s_{A 1}, s_{A 2}, \ldots, s_{A \mu_{A}}\right)$, and write:

$$
\mathbf{A}=\mathbf{A}\left(\mathbf{s}_{\mathbf{A}}\right)=\mathbf{A}\left(s_{A 1}, s_{A 2}, \ldots, s_{A \mu_{A}}\right) .
$$

Similarly, we denote the matrices $\mathbf{B}, \mathbf{C}$ and $\mathbf{D}$ as:

$$
\begin{aligned}
& \mathbf{B}=\mathbf{B}\left(\mathbf{s}_{\mathbf{B}}\right)=\mathbf{B}\left(s_{B 1}, s_{B 2}, \ldots, s_{B \mu_{B}}\right) \\
& \mathbf{C}=\mathbf{C}\left(\mathbf{s}_{\mathbf{C}}\right)=\mathbf{C}\left(s_{C 1}, s_{C 2}, \ldots, s_{C \mu_{C}}\right) \\
& \mathbf{D}=\mathbf{D}\left(\mathbf{s}_{\mathbf{D}}\right)=\mathbf{D}\left(s_{D 1}, s_{D 2}, \ldots, s_{D \mu_{D}}\right)
\end{aligned}
$$

For simplicity of notation, we sometimes write, for example, $\mathbf{A}_{\mathbf{s}_{\mathbf{A}}}$ to represent $\mathbf{A}\left(\mathbf{s}_{\mathbf{A}}\right)$.

Recall that the matrix $\overline{\mathbf{A}}$ is derived from $\mathbf{A}$ by replacing each variable $s_{A i}$, for $1 \leq i \leq \mu_{A}$, by its conjugate. Denote the conjugate of each variable by $s_{A i}^{*}$, for $1 \leq i \leq \mu_{A}$, and denote the vector of these conjugates by $\mathbf{s}_{\mathbf{A}}{ }^{*}$. Now, we write:

$$
\overline{\mathbf{A}}=\mathbf{A}\left(\mathbf{s}_{\mathbf{A}}{ }^{*}\right)=\mathbf{A}\left(s_{A 1}^{*}, s_{A 2}^{*}, \ldots, s_{A \mu_{A}}^{*}\right)
$$

and similarly for $\overline{\mathbf{B}}, \overline{\mathbf{C}}$, and $\overline{\mathbf{D}}$.

We state that a matrix $\mathbf{X}\left(\mathbf{s}_{\mathbf{X}}\right)$ is of similar form to a matrix $\mathbf{Y}\left(\mathbf{s}_{\mathbf{Y}}\right)$ (or just $\mathbf{X}$ is of similar form to $\mathbf{Y}$, for short) if $\mathbf{X}=$ $k_{X} \mathbf{Y}\left(\mathbf{s}_{\mathbf{X}}\right)$, where $\mathbf{s}_{\mathbf{X}}$ is a vector containing distinct complex variables $s_{X 1}, s_{X 2}, \ldots, s_{X \mu_{X}}$, and similarly, $\mathbf{s}_{\mathbf{Y}}$ is a vector containing distinct complex variables $s_{Y 1}, s_{Y 2}, \ldots, s_{Y \mu_{Y}}$, and $k_{X}$ is an arbitrary, non-zero, real coefficient. In this notation, we stipulate that the number of variables $\mu_{X}$ in $\mathbf{X}$ is at most equal to the number of variables $\mu_{Y}$ in $\mathbf{Y}$. To illustrate an example with $\mu_{X}=\mu_{Y}=2, \mathbf{X}\left(\mathbf{s}_{\mathbf{X}}\right)=\left[\begin{array}{cc}s_{X 1} & s_{X 2} \\ -s_{X 2}^{*} & s_{X 1}^{*}\end{array}\right]$ (which presents the Alamouti code with two variables) is of similar form to $\mathbf{Y}\left(\mathbf{s}_{\mathbf{Y}}\right)=\left[\begin{array}{cc}s_{Y 1} & s_{Y 2} \\ -s_{Y 2}^{*} & s_{Y 1}^{*}\end{array}\right]$, since $\mathbf{X}=$ $\mathbf{Y}\left(\mathbf{s}_{\mathbf{X}}\right)=\mathbf{Y}\left(s_{X 1}, s_{X 2}\right)$. To illustrate the case where $\mu_{X}=1$ and $\mu_{Y}=2, \mathbf{X}\left(\mathbf{s}_{\mathbf{X}}\right)=\left[\begin{array}{cc}s_{X 1} & s_{X 1} \\ -s_{X 1}^{*} & s_{X 1}^{*}\end{array}\right]$ (which presents the Alamouti code with only one variable) is also of similar form to $\mathbf{Y}$ since $\mathbf{X}=\mathbf{Y}\left(\mathbf{s}_{\mathbf{X}}\right)=\mathbf{Y}\left(s_{X 1}\right)$.

By this notation, when we state that the matrix $\mathbf{C}$ in (10) is of similar form to the matrix $\mathbf{B}$, for instance, we imply that $\mathbf{C}$ can be represented as $\mathbf{C}=k_{C} \mathbf{B}\left(\mathbf{s}_{\mathbf{C}}\right)$ where the number of complex variables $\mu_{C}$ in $\mathbf{C}$ is at most equal to the number of complex variables $\mu_{B}$ in $\mathbf{B}$, i.e., $\mu_{C} \leq \mu_{B}$.

Finally, we denote $\mathbf{I}_{n}$ to be an identity matrix of order $n$.

\section{Design Methods}

In this section, we provide two new methods to construct square CO STBCs. In each case, we use sub-matrices of order $n$ to build CO STBCs of order $N=4 n$. Our methods generalize the Williamson and Wallis-Whiteman arrays, which were originally used to build real orthogonal designs [11] (pp. 121 and 99 , respectively).

Theorem 1: If the sub-matrices $\mathbf{A}, \mathbf{B}, \mathbf{C}$ and $\mathbf{D}$ of order $n$ satisfy the following necessary conditions:

1) A, B, C, and $\mathbf{D}$ are orthogonal themselves and:

$$
\mathbf{A}^{H} \mathbf{A}+\mathbf{B}^{H} \mathbf{B}+\mathbf{C}^{H} \mathbf{C}+\mathbf{D}^{H} \mathbf{D}=\sum_{i \in \mathcal{I}_{U}} l_{i}\left|s_{i}\right|^{2} \mathbf{I}_{n}
$$

where $l_{i}$ are definitely positive, real coefficients, and the complex variables $s_{i}$ may be in $U_{1}, U_{2}, U_{3}$ or $U_{4}$ which are defined in (7).

2) The matrices $\mathcal{O}^{\prime}=\left[\begin{array}{cc}\mathbf{A} & \mathbf{B} \\ -\mathbf{B} & \mathbf{A}\end{array}\right]$ and $\mathcal{O}^{\prime \prime}=$ $\left[\begin{array}{cc}\mathbf{A} & \overline{\mathbf{B}} \\ -\overline{\mathbf{B}} & \mathbf{A}\end{array}\right]$ are square Complex Orthogonal Designs

3) $\mathbf{B}_{\mathbf{s}}^{H} \mathbf{B}_{\mathbf{s}^{\prime}}$ and $\mathbf{B}_{\mathbf{s}}^{T} \mathbf{B}_{\mathbf{s}^{\prime}}$ are symmetric for any possible pair of vectors $\mathbf{s}$ and $\mathbf{s}^{\prime}$ of complex variables, where $\mathbf{B}_{\mathbf{s}}$ and $\mathbf{B}_{\mathbf{s}^{\prime}}$ are shorthand for $\mathbf{B}(\mathbf{s})$ and $\mathbf{B}\left(\mathbf{s}^{\prime}\right)$, respectively.

4) $\mathbf{C}$ and $\mathbf{D}$ are of similar form to $\mathbf{B}$ and $\mathbf{B}$, respectively, $\overline{\mathbf{B}}$ and $\mathbf{B}$, respectively, $\mathbf{B}$ and $\overline{\mathbf{B}}$ respectively, or $\overline{\mathbf{B}}$ and $\overline{\mathbf{B}}$, respectively, i.e., $\mathbf{C}$ and $\mathbf{D}$ can be presented as one of the following forms:

$$
\begin{aligned}
& \left\{\begin{array} { l } 
{ \mathbf { C } = k _ { C } \mathbf { B } ( \mathbf { s } _ { \mathbf { C } } ) } \\
{ \mathbf { D } = k _ { D } \mathbf { B } ( \mathbf { s } _ { \mathbf { D } } ) }
\end{array} \quad \left\{\begin{array}{l}
\mathbf{C}=k_{C} \mathbf{B}\left(\mathbf{s}_{\mathbf{C}}^{*}\right) \\
\mathbf{D}=k_{D} \mathbf{B}\left(\mathbf{s}_{\mathbf{D}}\right)
\end{array}\right.\right. \\
& \left\{\begin{array} { l } 
{ \mathbf { C } = k _ { C } \mathbf { B } ( \mathbf { s } _ { \mathbf { C } } ) } \\
{ \mathbf { D } = k _ { D } \mathbf { B } ( \mathbf { s } _ { \mathbf { D } } ^ { * } ) }
\end{array} \quad \left\{\begin{array}{l}
\mathbf{C}=k_{C} \mathbf{B}\left(\mathbf{s}_{\mathbf{C}}^{*}\right) \\
\mathbf{D}=k_{D} \mathbf{B}\left(\mathbf{s}_{\mathbf{D}}^{*}\right)
\end{array}\right.\right.
\end{aligned}
$$

where $k_{C}$ and $k_{D}$ are arbitrary (positive or negative), real coefficients, and $\mu_{C} \leq \mu_{B}, \mu_{D} \leq \mu_{B}$ then

$$
\mathcal{O}=\left[\begin{array}{cccc}
\mathbf{A} & \mathbf{B} & \mathbf{C} & \mathbf{D} \\
-\mathbf{B} & \mathbf{A} & \overline{\mathbf{D}} & -\overline{\mathbf{C}} \\
-\mathbf{C} & -\overline{\mathbf{D}} & \mathbf{A} & \overline{\mathbf{B}} \\
-\mathbf{D} & \overline{\mathbf{C}} & -\overline{\mathbf{B}} & \mathbf{A}
\end{array}\right]
$$

is a $\mathrm{CO} \mathrm{STBC}$ of order $N=4 n$. If all coefficients $l_{i}=1$, for $i \in \mathcal{I}_{U}$, then $\mathcal{O}$ is called square CO STBC without Linear Processing (LP) (or just square CO STBC for short). Otherwise, $\mathcal{O}$ is considered as a square CO STBC with LP. If $\left(\mu_{A}+\mu_{B}+\mu_{C}+\mu_{D}\right)=\mu(N)$, then $\mathcal{O}$ is a square, maximum rate CO STBC of order $4 n$.

Proof: We prove Theorem 1 for the case that $\mathbf{C}$ and $\mathbf{D}$ are of similar form to $\mathbf{B}$ and $\mathbf{B}$, respectively. Similar arguments can be applied to three other cases. From (13), we have Equation (14), where $\mathcal{L}$ in the matrix $\mathbf{M}$ denotes the lower triangular part under the main diagonal whose elements are the Hermitian transposes of the corresponding elements in the upper triangular part. For instance, we have the element $\mathcal{L}(2,1)=\mathbf{B}^{H} \mathbf{A}-\mathbf{A}^{H} \mathbf{B}+\overline{\mathbf{D}}^{H} \mathbf{C}-\overline{\mathbf{C}}^{H} \mathbf{D}$.

First, we prove the following equalities:

$$
\begin{aligned}
\overline{\mathbf{B}}^{H} \overline{\mathbf{B}} & =\mathbf{B}^{H} \mathbf{B} \\
\overline{\mathbf{C}}^{H} \overline{\mathbf{C}} & =\mathbf{C}^{H} \mathbf{C} \\
\overline{\mathbf{D}}^{H} \overline{\mathbf{D}} & =\mathbf{D}^{H} \mathbf{D}
\end{aligned}
$$

Since $\mathbf{B}$ is orthogonal, we have:

$$
\mathbf{B}^{H} \mathbf{B}=\mathbf{B B}^{H}=\sum_{i \in \mathcal{I}_{U_{2}}} l_{i}\left|s_{i}\right|^{2} \mathbf{I}_{n}
$$

which implies that $\mathbf{B}^{H} \mathbf{B}$ is a real, diagonal matrix and therefore:

$$
\mathbf{B}^{H} \mathbf{B}=\left[\left(\mathbf{B}^{H} \mathbf{B}\right)^{T}\right]^{H}
$$

Using Eq. (18), it follows that:

$$
\mathbf{B}^{H} \mathbf{B}=\left[\mathbf{B}^{T} \overline{\mathbf{B}}\right]^{H}=\overline{\mathbf{B}}^{H}\left(\mathbf{B}^{T}\right)^{H}=\overline{\mathbf{B}}^{H} \overline{\mathbf{B}}
$$

Therefore, (15) has been proved. The same arguments can be applied to prove (16) and (17). Hence, if $\mathbf{A}, \mathbf{B}, \mathbf{C}$ and $\mathbf{D}$ are orthogonal themselves and satisfy (11), then all elements (i.e. 


$$
\begin{aligned}
\mathbb{M}= & \mathcal{O}^{H} \mathcal{O} \\
= & {\left[\begin{array}{cc}
A^{H} A+B^{H} B+C^{H} C+D^{H} D & A^{H} B-B^{H} A+C^{H} \bar{D}-D^{H} \bar{C} \\
& A^{H} A+B^{H} B+\bar{C}^{H} \bar{C}+\bar{D}^{H} \bar{D}
\end{array}\right.} \\
\mathcal{L} &
\end{aligned}
$$

$A^{H} C-C^{H} A-B^{H} \bar{D}+D^{H} \bar{B}$
$B^{H} C-\bar{C}^{H} \bar{B}+A^{H} \bar{D}-\bar{D}^{H} A$
$A^{H} A+\bar{B}^{H} \bar{B}+C^{H} C+\bar{D}^{H} \bar{D}$

$\left.\begin{array}{l}A^{H} D-D^{H} A+B^{H} \bar{C}-C^{H} \bar{B} \\ B^{H} D-\bar{D}^{H} \bar{B}-A^{H} \bar{C}+\bar{C}^{H} A \\ C^{H} D-\bar{D}^{H} \bar{C}+A^{H} \bar{B}-\bar{B}^{H} A \\ A^{H} A+\bar{B}^{H} \bar{B}+\bar{C}^{H} \bar{C}+D^{H} D\end{array}\right]$

sub-matrices) on the main diagonal of the matrix $\mathbf{M}=\mathcal{O}^{H} \mathcal{O}$ are equal to:

$$
\mathbf{A}^{H} \mathbf{A}+\mathbf{B}^{H} \mathbf{B}+\mathbf{C}^{H} \mathbf{C}+\mathbf{D}^{H} \mathbf{D}=\sum_{i \in \mathcal{I}_{U}} l_{i}\left|s_{i}\right|^{2} \mathbf{I}_{n}
$$

Second, we prove the following equalities:

$$
\begin{aligned}
\mathbf{A}^{H} \mathbf{B}-\mathbf{B}^{H} \mathbf{A} & =\mathbf{O}_{n} \\
\mathbf{A}^{H} \mathbf{C}-\mathbf{C}^{H} \mathbf{A} & =\mathbf{O}_{n} \\
\mathbf{A}^{H} \mathbf{D}-\mathbf{D}^{H} \mathbf{A} & =\mathbf{O}_{n}
\end{aligned}
$$

where $\mathbf{O}_{n}$ is a zero matrix of order $n$. Eq. (19) holds as $\mathcal{O}^{\prime}$ is a COD. Additionally, because $\mathbf{C}$ and $\mathbf{D}$ are of similar form to B (see (12)), the equalities (20) and (21) are straightforwardly proved (multiplication with real coefficients $k_{C}$ and $k_{D}$ does not change the property (19)).

Third, we prove the following equalities:

$$
\begin{aligned}
\mathbf{B}^{H} \overline{\mathbf{C}}-\mathbf{C}^{H} \overline{\mathbf{B}} & =\mathbf{O}_{n} \\
\mathbf{B}^{H} \overline{\mathbf{D}}-\mathbf{D}^{H} \overline{\mathbf{B}} & =\mathbf{O}_{n} \\
\mathbf{C}^{H} \overline{\mathbf{D}}-\mathbf{D}^{H} \overline{\mathbf{C}} & =\mathbf{O}_{n}
\end{aligned}
$$

Since $\mathbf{B}_{\mathbf{s}}^{T} \mathbf{B}_{\mathbf{s}^{\prime}}$ is symmetric for any pair of vectors $\mathbf{s}$ and $\mathbf{s}^{\prime}$ of complex variables, it follows that $\left(\mathbf{B}_{\mathbf{s}}^{T} \mathbf{B}_{\mathbf{s}^{\prime}}\right)^{H} \equiv \mathbf{B}_{\mathbf{s}^{\prime}}^{H} \mathbf{B}_{\mathbf{s}}^{H^{T}}$ is also symmetric. Using this symmetry, it follows that:

$$
\begin{aligned}
\mathbf{B}_{\mathbf{s}^{\prime}}^{H} \mathbf{B}_{\mathbf{s}}^{H^{T}}=\left[\mathbf{B}_{\mathbf{s}^{\prime}}^{H} \mathbf{B}_{\mathbf{s}}^{H^{T}}\right]^{T} & \Leftrightarrow \quad \mathbf{B}_{\mathbf{s}^{\prime}}^{H} \overline{\mathbf{B}}_{\mathbf{s}}=\mathbf{B}_{\mathbf{s}}^{H} \mathbf{B}_{\mathbf{s}^{\prime}}^{H^{T}} \\
& \Leftrightarrow \quad \mathbf{B}_{\mathbf{s}^{\prime}}^{H} \overline{\mathbf{B}}_{\mathbf{s}}=\mathbf{B}_{\mathbf{s}}^{H} \overline{\mathbf{B}}_{\mathbf{s}^{\prime}}
\end{aligned}
$$

In other words, we have:

$$
\mathbf{B}_{\mathbf{s}^{\prime}}^{H} \overline{\mathbf{B}}_{\mathbf{s}}-\mathbf{B}_{\mathbf{s}}^{H} \overline{\mathbf{B}}_{\mathbf{s}^{\prime}}=\mathbf{O}_{n}
$$

for any pair of vectors $\mathbf{s}$ and $\mathbf{s}^{\prime}$. Due to the fact that $\mathbf{C}$ and $\mathbf{D}$ are of similar form to $\mathbf{B}$, by replacing $\mathbf{B}_{\mathbf{s}}$ and $\mathbf{B}_{\mathbf{s}^{\prime}}$ in (25) by $\mathbf{B}, \mathbf{C}$ or $\mathbf{D}$, the equalities (22), (23) and (24) are proved.

From (19)-(24), we see that the elements $\mathbf{M}(1,2), \mathbf{M}(1,3)$ and $\mathbf{M}(1,4)$ of the matrix $\mathbf{M}=\mathcal{O}^{H} \mathcal{O}$ are zero matrices.

Fourth, we prove the following equalities:

$$
\begin{aligned}
\mathbf{B}^{H} \mathbf{C}-\overline{\mathbf{C}}^{H} \overline{\mathbf{B}} & =\mathbf{O}_{n} \\
\mathbf{B}^{H} \mathbf{D}-\overline{\mathbf{D}}^{H} \overline{\mathbf{B}} & =\mathbf{O}_{n} \\
\mathbf{C}^{H} \mathbf{D}-\overline{\mathbf{D}}^{H} \mathbf{C} & =\mathbf{O}_{n}
\end{aligned}
$$

Due to $\mathbf{B}_{\mathbf{s}}^{H} \mathbf{B}_{\mathbf{s}^{\prime}}$ being symmetric, the following equalities hold:

$$
\begin{aligned}
& \mathbf{B}_{\mathbf{s}}^{H} \mathbf{B}_{\mathbf{s}^{\prime}}=\left[\mathbf{B}_{\mathbf{s}}^{H} \mathbf{B}_{\mathbf{s}^{\prime}}\right]^{T} \Leftrightarrow \mathbf{B}_{\mathbf{s}}^{H} \mathbf{B}_{\mathbf{s}^{\prime}}=\mathbf{B}_{\mathbf{s}^{\prime}}^{T} \overline{\mathbf{B}}_{\mathbf{s}} \\
& \Leftrightarrow \quad \mathbf{B}_{\mathbf{s}}^{H} \mathbf{B}_{\mathbf{s}^{\prime}}=\overline{\mathbf{B}}_{\mathbf{s}^{\prime}}^{H} \overline{\mathbf{B}}_{\mathbf{s}} \Leftrightarrow \mathbf{B}_{\mathbf{s}}^{H} \mathbf{B}_{\mathbf{s}^{\prime}}-\overline{\mathbf{B}}_{\mathbf{s}^{\prime}}^{H} \overline{\mathbf{B}}_{\mathbf{s}}=\mathbf{O}_{n}
\end{aligned}
$$

for any pair of vectors $\mathbf{s}$ and $\mathbf{s}^{\prime}$. Due to $\mathbf{C}$ and $\mathbf{D}$ being of similar form to $\mathbf{B}$, by replacing $\mathbf{B}_{\mathbf{s}}$ and $\mathbf{B}_{\mathbf{s}^{\prime}}$ in (29) by $\mathbf{B}, \mathbf{C}$ or $\mathrm{D}$, the equalities (26)-(28) are proved.

Finally, we prove that:

$$
\begin{aligned}
\mathbf{A}^{H} \overline{\mathbf{B}}-\overline{\mathbf{B}}^{H} \mathbf{A} & =\mathbf{O}_{n} \\
\mathbf{A}^{H} \overline{\mathbf{C}}-\overline{\mathbf{C}}^{H} \mathbf{A} & =\mathbf{O}_{n} \\
\mathbf{A}^{H} \overline{\mathbf{D}}-\overline{\mathbf{D}}^{H} \mathbf{A} & =\mathbf{O}_{n}
\end{aligned}
$$

Eq. (30) holds since $\mathcal{O}^{\prime \prime}$ is a COD. Because $\mathbf{C}$ and $\mathbf{D}$ are of similar form to $\mathbf{B}$, by replacing $\mathbf{B}$ in (30) by $\mathbf{C}$ or $\mathbf{D}$, the equalities (31) and (32) are proved.
From (26)-(28) and (30)-(32), it follows that the elements $\mathbf{M}(2,3)=\mathbf{M}(2,4)=\mathbf{M}(3,4)=\mathbf{O}_{n}$. Since the lower triangular part $\mathcal{L}$ is the Hermitian transpose of the upper part, all elements in $\mathcal{L}$ are also zero matrices. Hence, $M$ can be presented as:

$$
\mathbf{M}=\sum_{i \in \mathcal{I}_{U}} l_{i}\left|s_{i}\right|^{2} \operatorname{diag}\left(\mathbf{I}_{n}, \mathbf{I}_{n}, \mathbf{I}_{n}, \mathbf{I}_{n}\right)=\sum_{i \in \mathcal{I}_{U}} l_{i}\left|s_{i}\right|^{2} \mathbf{I}_{N}
$$

where diag denotes a diagonal matrix. In other words, the matrix $\mathcal{O}$ in (13) is a square COD (also CO STBC) of order $N=4 n$ with $\left(\mu_{A}+\mu_{B}+\mu_{C}+\mu_{D}\right)$ variables. Note that, if $\mathcal{O}$ comprises the maximum number of variables, i.e., Eq. (9) is satisfied, then $\mathcal{O}$ is a square, maximum rate $\mathrm{CO}$ STBC of order $4 n$. Theorem 1 has been proved.

Similarly, we derived the following theorem, which is a variation of the Wallis-Whiteman array [11] (pp. 99), modified to apply to complex matrices:

Theorem 2: If the sub-matrices $\mathbf{A}, \mathbf{B}, \mathbf{C}$ and $\mathbf{D}$ of order $n$ satisfy the following necessary conditions:

1) A, B, C and $\mathbf{D}$ are orthogonal themselves and:

$$
\mathbf{A}^{H} \mathbf{A}+\mathbf{B}^{H} \mathbf{B}+\mathbf{C}^{H} \mathbf{C}+\mathbf{D}^{H} \mathbf{D}=\sum_{i \in \mathcal{I}_{U}} l_{i}\left|s_{i}\right|^{2} \mathbf{I}_{n}
$$

where $l_{i}$ are definitely positive, real coefficients, and the complex variables $s_{i}$ may be in $U_{1}, U_{2}, U_{3}$ or $U_{4}$, which are defined in (7).

2) The matrices $\mathcal{O}^{\prime}=\left[\begin{array}{cc}\mathbf{C} & \mathbf{A} \\ -\mathbf{A} & \mathbf{C}\end{array}\right]$ and $\mathcal{O}^{\prime \prime}=$ $\left[\begin{array}{cc}\mathbf{C} & \overline{\mathbf{A}} \\ -\overline{\mathbf{A}} & \mathbf{C}\end{array}\right]$ are square Complex Orthogonal Designs (COD) of order $2 n$.

3) $\mathbf{A}_{\mathbf{s}}^{H} \mathbf{A}_{\mathbf{s}^{\prime}}$ and $\mathbf{A}_{\mathbf{s}}^{T} \mathbf{A}_{\mathbf{s}^{\prime}}$ are symmetric for any possible pair of vectors $\mathbf{s}$ and $\mathbf{s}^{\prime}$ of complex variables, where $\mathbf{A}_{\mathbf{s}}$ and $\mathbf{A}_{\mathbf{s}^{\prime}}$ are shorthand for $\mathbf{A}(\mathbf{s})$ and $\mathbf{A}\left(\mathbf{s}^{\prime}\right)$, respectively.

4) $\mathbf{B}$ and $\mathbf{D}$ are of similar form to $\mathbf{A}$ and $\mathbf{A}$, respectively, $\overline{\mathbf{A}}$ and $\mathbf{A}$, respectively, $\mathbf{A}$ and $\overline{\mathbf{A}}$ respectively, or $\overline{\mathbf{A}}$ and $\overline{\mathbf{A}}$, respectively, i.e., $\mathbf{B}$ and $\mathbf{D}$ can be presented as one of the following forms:

$$
\begin{aligned}
& \left\{\begin{array} { l } 
{ \mathbf { B } = k _ { B } \mathbf { A } ( \mathbf { s } _ { \mathbf { B } } ) } \\
{ \mathbf { D } = k _ { D } \mathbf { A } ( \mathbf { s } _ { \mathbf { D } } ) }
\end{array} \quad \left\{\begin{array}{l}
\mathbf{B}=k_{B} \mathbf{A}\left(\mathbf{s}_{\mathbf{B}}^{*}\right) \\
\mathbf{D}=k_{D} \mathbf{A}\left(\mathbf{s}_{\mathbf{D}}\right)
\end{array}\right.\right. \\
& \left\{\begin{array} { l } 
{ \mathbf { B } = k _ { B } \mathbf { A } ( \mathbf { s } _ { \mathbf { B } } ) } \\
{ \mathbf { D } = k _ { D } \mathbf { A } ( \mathbf { s } _ { \mathbf { D } } ^ { * } ) }
\end{array} \quad \left\{\begin{array}{l}
\mathbf{B}=k_{B} \mathbf{A}\left(\mathbf{s}_{\mathbf{B}}^{*}\right) \\
\mathbf{D}=k_{D} \mathbf{A}\left(\mathbf{s}_{\mathbf{D}}^{*}\right)
\end{array}\right.\right.
\end{aligned}
$$

where $k_{B}$ and $k_{D}$ are arbitrary (positive or negative), real coefficients, and $\mu_{B} \leq \mu_{A}, \mu_{D} \leq \mu_{A}$ then

$$
\mathcal{O}=\left[\begin{array}{cccc}
\mathbf{A} & \mathbf{B} & \mathbf{C} & \mathbf{D} \\
-\overline{\mathbf{B}} & \overline{\mathbf{A}} & -\mathbf{D} & \mathbf{C} \\
-\mathbf{C} & \overline{\mathbf{D}} & \mathbf{A} & -\overline{\mathbf{B}} \\
-\overline{\mathbf{D}} & -\mathbf{C} & \mathbf{B} & \overline{\mathbf{A}}
\end{array}\right]
$$

is a CO STBC of order $N=4 n$. If all coefficients $l_{i}=1$ for $i \in \mathcal{I}_{U}$, then $\mathcal{O}$ is called square $\mathrm{CO}$ STBC without Linear Processing (LP) (or just square CO STBC for short). Otherwise, $\mathcal{O}$ is considered as a square CO STBC with LP. If $\left(\mu_{A}+\mu_{B}+\mu_{C}+\mu_{D}\right)=\mu(N)$, then $\mathcal{O}$ is a square, maximum rate CO STBC of order $4 n$.

Proof: The proof of Theorem 2 is similar to the proof of Theorem 1. 


\section{EXamples of MaXimum Rate, SQuare, Order-8 CO STBCS WITH NO ZERO ENTRIES}

In order to construct $8 \times 8 \mathrm{CO}$ STBCs of maximum rates using the proposed methods in Theorems 1 and 2, the main task is to find two $2 \times 2$ sub-matrices which satisfy certain properties. This is easier than finding eight $8 \times 8$ weighting matrices simultaneously as in the AOD approach [10].

Using Theorem 1 and Theorem 2, we construct here some square CO STBCs of order $N=8$ (with or without LP) with the maximum number of variables $\mu(8)=4$. The sub-matrices $\mathbf{A}$, $\mathbf{B}, \mathbf{C}, \mathbf{D}$ are of order $n=2$ and each sub-matrix comprises one variable. From Theorem 1 (correspondingly, Theorem 2), it is clear that the most crucial task for constructing square $\mathrm{CO}$ STBCs of order $4 n$ in our proposed methods is to find two matrices $\mathbf{A}$ and $\mathbf{B}$ (A and $\mathbf{C}$ ) satisfying the properties (2) and (3) in Theorem 1 (Theorem 2). We realize that various matrices $\mathbf{A}, \mathbf{B}, \mathbf{C}$, and $\mathbf{D}$ can satisfy those conditions, and derive here some of those cases for illustration.

Example 1: The following sub-matrices satisfy Theorem 1:

$$
\begin{aligned}
\mathbf{A} & =k_{1}\left[\begin{array}{cc}
s_{1} & s_{1} \\
-s_{1}^{*} & s_{1}^{*}
\end{array}\right] ; \mathbf{B}=k_{2}\left[\begin{array}{cc}
-s_{2}^{*} & s_{2}^{*} \\
s_{2} & s_{2}
\end{array}\right] \\
\mathbf{C} & =k_{3}\left[\begin{array}{cc}
-s_{3}^{*} & s_{3}^{*} \\
s_{3} & s_{3}
\end{array}\right] ; \mathbf{D}=k_{4}\left[\begin{array}{cc}
-s_{4}^{*} & s_{4}^{*} \\
s_{4} & s_{4}
\end{array}\right]
\end{aligned}
$$

for any real coefficients $k_{i},(i=1 \ldots 4)$.

In this example, $\mathbf{A}$ is a variation of the Alamouti code with only one variable, while $\mathbf{C}$ and $\mathbf{D}$ are each of similar form to $\mathbf{B}$. Then, $\mathcal{O}$ in (13) satisfies $\mathcal{O}^{H} \mathcal{O}=2 \sum_{i=1}^{4} k_{i}^{2}\left|s_{i}\right|^{2} \mathbf{I}_{8}$ and, consequently, $\mathcal{O}$ is a maximum rate, square, order-8 $\mathrm{CO}$ STBC (with or without LP depending on $k_{i}$ ). If $k_{i}=1$, for $i=1, \ldots, 4$, from (13), we have the following code:

$\left[\begin{array}{rrrrrrrr}s_{1} & s_{1} & -s_{2}^{*} & s_{2}^{*} & -s_{3}^{*} & s_{3}^{*} & -s_{4}^{*} & s_{4}^{*} \\ -s_{1}^{*} & s_{1}^{*} & s_{2} & s_{2} & s_{3} & s_{3} & s_{4} & s_{4} \\ s_{2}^{*} & -s_{2}^{*} & s_{1} & s_{1} & -s_{4} & s_{4} & s_{3} & -s_{3} \\ -s_{2} & -s_{2} & -s_{1}^{*} & s_{1}^{*} & s_{4}^{*} & s_{4}^{*} & -s_{3}^{*} & -s_{3}^{*} \\ s_{3}^{*} & -s_{3}^{*} & s_{4} & -s_{4} & s_{1} & s_{1} & -s_{2} & s_{2} \\ -s_{3} & -s_{3} & -s_{4}^{*} & -s_{4}^{*} & -s_{1}^{*} & s_{1}^{*} & s_{2}^{*} & s_{2}^{*} \\ s_{4}^{*} & -s_{4}^{*} & -s_{3} & s_{3} & s_{2} & -s_{2} & s_{1} & s_{1} \\ -s_{4} & -s_{4} & s_{3}^{*} & s_{3}^{*} & -s_{2}^{*} & -s_{2}^{*} & -s_{1}^{*} & s_{1}^{*}\end{array}\right]$
Examples with various other structures are given below.

Example 2: This example using Theorem 1 shows that the CO STBC G8 in (4) can be (indirectly) derived from our proposed methods. Let:

$$
\begin{aligned}
\mathbf{A} & =k_{1}\left[\begin{array}{cc}
s_{1}^{*} & s_{1}^{*} \\
s_{1} & -s_{1}
\end{array}\right] ; \mathbf{B}=k_{2}\left[\begin{array}{cc}
s_{2} & -s_{2} \\
s_{2}^{*} & s_{2}^{*}
\end{array}\right] \\
\mathbf{C} & =k_{3}\left[\begin{array}{cc}
s_{3} & -s_{3} \\
s_{3}^{*} & s_{3}^{*}
\end{array}\right] ; \mathbf{D}=k_{4}\left[\begin{array}{cc}
s_{4} & -s_{4} \\
s_{4}^{*} & s_{4}^{*}
\end{array}\right]
\end{aligned}
$$

In this example, $\mathbf{C}$ and $\mathbf{D}$ are each of similar form to $\mathbf{B}$. If $k_{i}=1$ for $i=1, \ldots, 4$, from (13), we have the following code:

$$
\left[\begin{array}{cccccccc}
s_{1}^{*} & s_{1}^{*} & s_{2} & -s_{2} & s_{3} & -s_{3} & s_{4} & -s_{4} \\
s_{1} & -s_{1} & s_{2}^{*} & s_{2}^{*} & s_{3}^{*} & s_{3}^{*} & s_{4}^{*} & s_{4}^{*} \\
-s_{2} & s_{2} & s_{1}^{*} & s_{1}^{*} & s_{4}^{*} & -s_{4}^{*} & -s_{3}^{*} & s_{3}^{*} \\
-s_{2}^{*} & -s_{2}^{*} & s_{1} & -s_{1} & s_{4} & s_{4} & -s_{3} & -s_{3} \\
-s_{3} & s_{3} & -s_{4}^{*} & s_{4}^{*} & s_{1}^{*} & s_{1}^{*} & s_{2}^{*} & -s_{2}^{*} \\
-s_{3}^{*} & -s_{3}^{*} & -s_{4} & -s_{4} & s_{1} & -s_{1} & s_{2} & s_{2} \\
-s_{4} & s_{4} & s_{3}^{*} & -s_{3}^{*} & -s_{2}^{*} & s_{2}^{*} & s_{1}^{*} & s_{1}^{*} \\
-s_{4}^{*} & -s_{4}^{*} & s_{3} & s_{3} & -s_{2} & -s_{2} & s_{1} & -s_{1}
\end{array}\right]
$$

We note that the CO STBC G8 in (4) can be derived from our CO STBC in (35) by multiplying every even row in (35) with $j$. However, G8 in (4) itself does not follow our proposed structure as the sub-matrices $\mathbf{A}$ and $\mathbf{B}$ in $\mathbf{G} 8$ do not satisfy the second condition in Theorem 1.

Example 3: This example illustrates the case in Theorem 2 where $\mathbf{B}$ and $\mathbf{D}$ are each of similar form to $\mathbf{A}$ :

$$
\begin{aligned}
\mathbf{A} & =k_{1}\left[\begin{array}{cc}
s_{1}^{*} & s_{1}^{*} \\
s_{1} & -s_{1}
\end{array}\right] ; \mathbf{B}=k_{2}\left[\begin{array}{cc}
s_{2}^{*} & s_{2}^{*} \\
s_{2} & -s_{2}
\end{array}\right] \\
\mathbf{C} & =k_{3}\left[\begin{array}{cc}
s_{3} & -s_{3} \\
s_{3}^{*} & s_{3}^{*}
\end{array}\right] ; \mathbf{D}=k_{4}\left[\begin{array}{cc}
s_{4}^{*} & s_{4}^{*} \\
s_{4} & -s_{4}
\end{array}\right]
\end{aligned}
$$

If $k_{i}=1$ for $i=1, \ldots, 4$, from (33), we have the following code:

$$
\left[\begin{array}{cccccccc}
s_{1}^{*} & s_{1}^{*} & s_{2}^{*} & s_{2}^{*} & s_{3} & -s_{3} & s_{4}^{*} & s_{4}^{*} \\
s_{1} & -s_{1} & s_{2} & -s_{2} & s_{3}^{*} & s_{3}^{*} & s_{4} & -s_{4} \\
-s_{2} & -s_{2} & s_{1} & s_{1} & -s_{4}^{*} & -s_{4}^{*} & s_{3} & -s_{3} \\
-s_{2}^{*} & s_{2}^{*} & s_{1}^{*} & -s_{1}^{*} & -s_{4} & s_{4} & s_{3}^{*} & s_{3}^{*} \\
-s_{3} & s_{3} & s_{4} & s_{4} & s_{1}^{*} & s_{1}^{*} & -s_{2} & -s_{2} \\
-s_{3}^{*} & -s_{3}^{*} & s_{4}^{*} & -s_{4}^{*} & s_{1} & -s_{1} & -s_{2}^{*} & s_{2}^{*} \\
-s_{4} & -s_{4} & -s_{3} & s_{3} & s_{2}^{*} & s_{2}^{*} & s_{1} & s_{1} \\
-s_{4}^{*} & s_{4}^{*} & -s_{3}^{*} & -s_{3}^{*} & s_{2} & -s_{2} & s_{1}^{*} & -s_{1}^{*}
\end{array}\right]
$$

All of the above codes are square, maximum rate CO STBCs of order $N=8$ with a full design, i.e., without any zeros for any complex signal constellations. The power is equally transmitted via each transmit antenna during every symbol time slot. For these reasons, the proposed CO STBCs are referred to as the improved, square CO STBCs.

\section{CONCLUSION}

By modifying the Williamson and Wallis-Whiteman arrays to apply to complex matrices, we have proposed two new methods of constructing square, order- $4 n$ CO STBCs from square, order- $n$ CO STBCs which satisfy certain properties as described in Theorems 1 and 2 .

Applying Theorems 1 and 2, we have constructed various square, maximum rate, order- $8 \mathrm{CO}$ STBCs with no zeros. In our $\mathrm{CO}$ STBCs, the transmitted symbols equally disperse through transmit antennas with the consequence that the power transmitted via each transmit antenna is equal during every symbol time slot. Additionally, our methods may be used to design square CO STBCs of order 16 or 32 from square $C O$ STBCs of order 4 or 8, respectively, provided that there exist sub-matrices satisfying the conditions of our theorems. The construction of square CO STBCs of higher orders, such as 16 or 32, requires further study, and this is our future work.

\section{REFERENCES}

[1] S. M. Alamouti, "A simple transmit diversity technique for wireless communications," IEEE J. Select. Areas Commun., vol. 16, no. 8, pp. $1451-1458$, Oct. 1998

[2] X.-B. Liang, "Orthogonal designs with maximal rates," IEEE Trans. Inform. Theory, vol. 49, no. 10, pp. 2468-2503, Oct. 2003

[3] V. Tarokh, H. Jafarkhani, and A. R. Calderbank, "Space-time block coding for wireless communications: performance results," IEEE J. Select. Areas Commun., vol. 17, no. 3, pp. 451 - 460, Mar. 1999.

[4] V. Tarokh, H. Jafarkhani, and A. R. Calderbank, "Space-time blocks codes from orthogonal designs," IEEE Trans. Inform. Theory, vol. 45, no. 5 , pp. 1456 - 1467 , July 1999

[5] O. Tirkkonen and A. Hottinen, "Square-matrix embeddable space-time blocks codes for complex signal constellations," IEEE Trans. Inform. Theory, vol. 48, no. 2, pp. 384 - 395, Feb. 2002.

[6] X.-B. Liang and X.-G. Xia, "On the nonexistence of rate-one generalized complex orthogonal designs," IEEE Trans. Inform. Theory, vol. 49, no. 11 , pp. $2984-2988$, Nov. 2003.

[7] L. C. Tran, J. Seberry, B. J. Wysocki, T. A. Wysocki, T. Xia, and Y. Zhao, "Two new complex orthogonal space-time codes for 8 transmit antennas," IEE Electronics Lett., vol. 40, no. 1, pp. 55-56, Jan. 2004.

[8] J. Seberry, L. C. Tran, Y. Wang, B. J. Wysocki, T. A. Wysocki, T. Xia, and Y. Zhao, "New complex orthogonal space-time block codes of order eight" in Signal Processing for Telecommunications and Multimedia, B. Honary T. A. Wysocki and B. J. Wysocki, Eds., vol. 27 of Multimedia systems and applications, pp. 173-182. Springer, New York, Oct. 2004.

[9] Y. Zhao, J. Seberry, T. Xia, Y. Wang, B. J. Wysocki, T. A. Wysocki, and L. C. Tran, "On amicable orthogonal designs of order 8 for complex space-time block codes," To appear in The Australasian Journal of Combinatorics (AJC), ISSN 1034-4942, 2005.

[10] C. Yuen, Y. L. Guan, and T. T. Tjhung, "Orthogonal space-time block code from amicable orthogonal design," Proc. IEEE. Int. Conf. Acoustic, Speech and Signal Processing ICASSP 2004, vol. 4, pp. 469-472, May 2004.

[11] A. V. Geramita and J. Seberry, Orthogonal designs: quadratic forms and Hadamard matrices, vol. 43, Lecture notes in pure and applied mathematics, Marcel Dekker, New York and Basel, 1979.

[12] G. Ganesan and P. Stoica, "Space-time diversity using orthogonal and amicable orthogonal designs," Proc. IEEE International Conference on Acoustics, Speech, and Signal Processing, vol. 5, pp. 2561-2564, June 2000 . 